\title{
Decision tree-based expert system for adverse drug reaction detection using fuzzy logic and genetic algorithm
}

\author{
Ayman M. Mansour* \\ Department of Communication, Electronics and Computer Engineering, Tafila Technical University, Tafila, Jordan
}

Received: 07-January-2018; Revised: 01-February-2018; Accepted: 26-April-2018

(C)2018 ACCENTS

\begin{abstract}
Early detection of unknown adverse drug reactions (ADRs) could save patient lives and prevent unnecessary hospitalizations. Current surveillance systems are not ideal for rapidly identifying rare unknown ADRs. Current methods largely rely on passive spontaneous reports, which suffer from serious underreporting, latency, and inconsistent reporting. A more effective system is needed as the electronic patient records become more and more easily accessible in various health organizations such as hospitals, medical centers and insurance companies. These data provide a new source of information that has great potentials to detect ADR signals much earlier. In this paper, we have developed a methodology that uses both decision tree and fuzzy logic to generate a decision model. The developed model is equipped with a fuzzy inference engine, which enables it to find the causal relationship between a drug and a potential ADR. This could assist healthcare professionals to early detect previously unknown ADRs. Optimizing fuzzy rule weights and fuzzy sets parameters using genetic algorithm has been embedded in the proposed system to achieve excellent performance and improve the accuracy of the developed model. To evaluate the performance of the system, we have implemented the system using Weka and FuzzyJess software packages, and generated simulation results. To conduct the experiments, clinical information on 280 patients treated at the Detroit Veterans Affairs Medical Center was used. Two physicians on the team independently reviewed the experiment results. Kappa statistics show excellent agreement between the physicians and the developed model.
\end{abstract}

\section{Keywords}

Adverse drug reactions, Fuzzy logic, Decision tree, Genetic algorithm.

\section{Introduction}

ADR was defined as "An appreciably harmful or unpleasant reaction. It results from an intervention related to the use of a medicinal product. Adverse effects usually predict hazard from future administration and warrant prevention, or specific treatment. It contributes to alternate the dosage regimen or withdrawal of the product" [1]. ADRs are a major public health problem in the USA [1, 2]. In 2014, for instance, adverse events reporting system which is managed by the USA that is food and drug administration (FDA) showed that 123,927 deaths were attributed to serious ADR and 807,270 serious cases were reported, which included, among others, hospitalization, life-threatening, and/or disability [2]. Figure 1 [2] illustrates the patient outcome(s) for ADRs since the year 2006 until the first quarter of 2015 .

*Author for correspondence
A complete understanding of the safe use of drugs is not possible at the time when the drug is developed or marketed. At that time, the safety information is only limited to a few thousand people in a typical clinical trials. For example, people were not aware of the risk of heart attacks associated with the use of rofecoxib until five years later after it was launched to the market.

Detecting ADR signal pairs is technically a complex problem [3-10]. This is the case if we realistically assume that there does not exist a set of rules that are readily acceptable to all human experts (e.g., physicians, epidemiologists and pharmacists). The parameters used in identifying the signal pairs are really a vague, subjective measure rather than an objective measure. Furthermore, human experts often disagree one another owing to their knowledge and experiences and there is no "ground truth" to indicate which physician is right or wrong. 


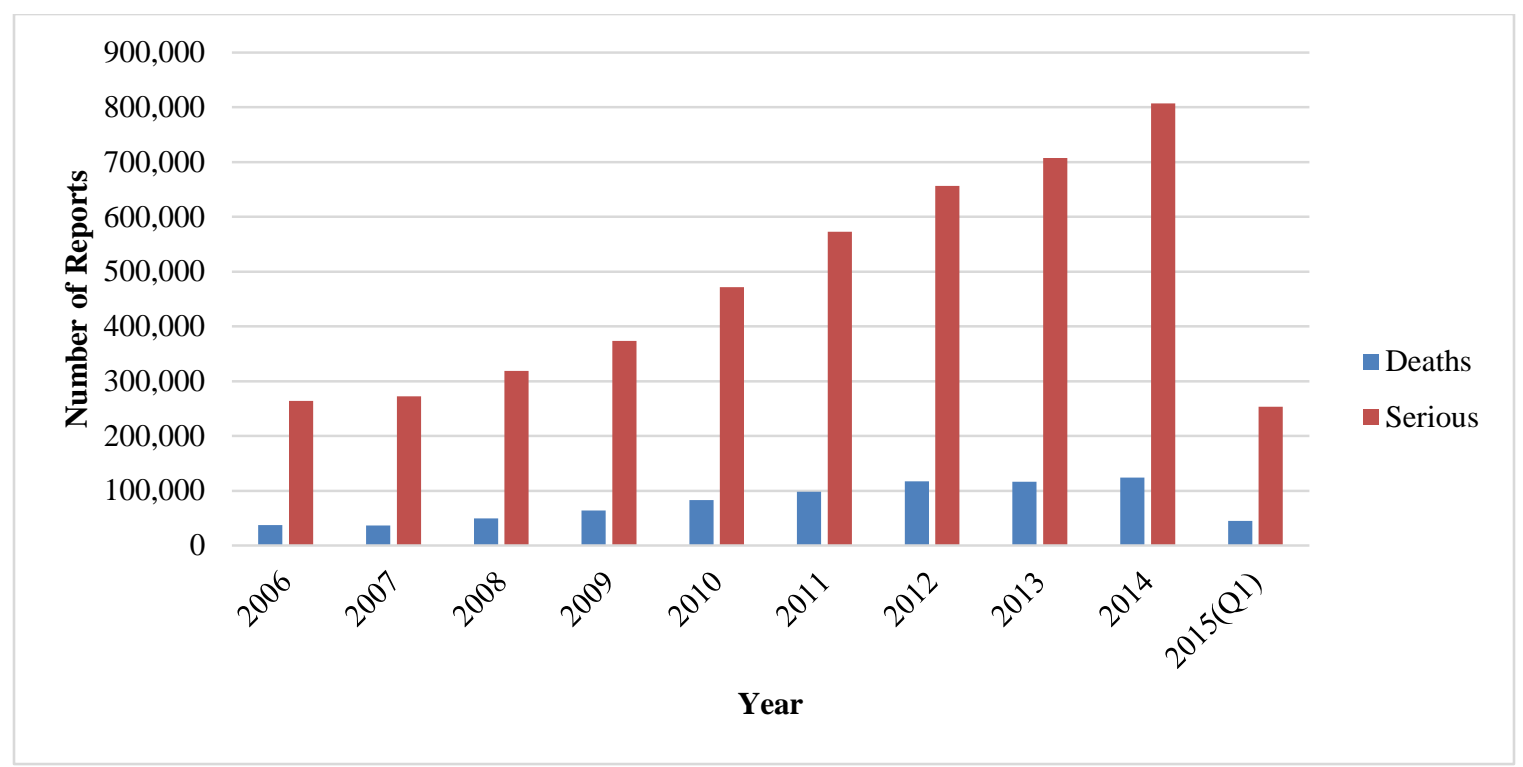

Figure 1 Patient outcome(s) for ADRs since the year 2006 until the first quarter of 2015 [2]

Given the limited information available when the drug is marketed, post-marketing study has become increasingly important. Post-marketing surveillance is the process of identifying, reporting, and responding to the issues occurred while taking medication [11-18]. This method is the principal method used for monitoring the safety of marketed drugs nowadays [19]. The responding includes actions that can be taken to improve product safety and protect the public health, such as labeling changes, safety alerts or product withdrawals $[3,12]$. Even if the report does suggest labeling changes, the information provided will be kept for further investigated especially when more information became available. Once the reports are studied and evaluated, the data generated can help to identify ADR with certain medications and investigate these ADRs to provide clear indicators that can be used to identify other ADR resulted from other medications. To date, many methods have been adopted into postmarketing studies, including ADR case review, comparative observational study, ADRs spontaneous reporting, and data mining algorithms.

The most important source of adverse event information to the FDA in USA is the MedWatch program [4]. In this program, health care providers and patients can submit an adverse event report via several mechanisms, including an online report form, phone, fax and mail. Detection of signals in MedWatch is limited due to the low percentage of adverse events which are rare events (less than 10\%). The quality of the reports and the accuracy of the information written in the reports can also affect the detection performance of the adverse events. Some time, for an instant, the health providers don't appreciate clinical finding or adverse events until the occurrence became wider in the patient population. Also, we need to remember that MedWatch reporting is voluntary job and many suspected adverse events are not reported based on the physician's point of view $[9,20]$.

Other spontaneous reporting systems, such as yellow card in the UK, suffer from low reporting rates, typically less than $10 \%$. Underreporting of ADRs is a common problem in spontaneous surveillance programs which can delay adverse event detection and underestimate a problem size [13-18]. Knowing the factors that may confirm an adverse event will assist heath providers and physicians in establishing ways to correct underreporting. Different reasons that need to be analyzed in order to improve the quality of reporting [21]. There are huge numbers of reports that are available in the databases. This makes it difficult for physicians to analyze the reports of adverse reactions. They have little time especially most of the reported events have a low likelihood of a causal relation.

Several data mining algorithms have been well described in the literature; some are based on simple analysis, e.g., the reporting odds ratios and the proportional reporting ratios [22, 23]. 
Data mining algorithms are being used to explore spontaneous reporting databases for adverse reaction signal pairs. The finding of data mining algorithms has been compared with those comes from classical reporting methods in [5]. Most adverse events identified by both methods highlight in the product labeling. Classical reporting methods identify four potentially unexpected serious adverse events which may lead to label changing and close monitoring. The other finding of that paper that none of these adverse events has been identified using the data mining algorithms. This is not helpful since it cannot detect or enhance the classical reporting methods, surveillance in this particular setting. Data mining algorithm's performance may be enhanced by selecting the most appropriate Pharmacovigilance tools that are designed specifically for each situation [24]. Also in [25] five data mining algorithms are used for identifying possible ADRs from spontaneous reports information. The study concludes that the detected drug-ADR signal pairs vary between different methodologies and this makes the data mining algorithms unreliable.

The availability of huge amounts of reported events, including false-positive signals as a result of the existence of confounding will produce unhelpful hypotheses. This may affect the capability of data mining algorithms to detect true positive signals of real causal associations. This will lead to serious consequences that delay the detection of the signal pairs.

Data mining algorithms had not been generally accepted by health providers, physicians or pharmacists. This is due to the three main reasons which are as follows [3]:

1. The apparent complexity of its mathematics and the hidden strategy of detecting the signal pairs deter those unfamiliar with statistics.

2. Even with the increasing availability of epidemiological and pharmacoepidemiological databases, background information for calculating prior probabilities is still either unclear or unavailable.

3. The pre-market data from clinical trials is usually not available for the public in order to be used for estimating the prior probability. The data has been kept confidential between the drug companies and regulatory authorities. Even when they are available, they may not be in a suitable format.

Since no consensus exists regarding the use of data mining algorithms, the use of such methods in
Pharmacovigilance is still limited and not embraced by health providers. Data mining algorithm may draw attention to more "surprising" drug-event signal pairs. In fact, the unknown features of the data (i.e., the event background incidences) and the underreporting problem of the events from health providers to the regulatory authorities will have direct effect the outcome of data mining algorithms [26].

A decision-tree based model is an effective supervised technique to implement the classification methods in high-dimensional data has been developed in this paper [27]. The developed system is equipped with an intelligent decision maker that uses a fuzzy rule-based reasoning capability. Fuzzy logic is used to represent, interpret, and compute vague and/or subjective information which is very common in medicine [28-30]. Fuzzy logic is commonly accepted by physicians more than other intelligent techniques because the fuzzy rules are extracted from domain experts, i.e. physicians. The fuzzy inference system is implemented using the freeware FuzzyJess [31].

The developed system is equipped with a fuzzy inference engine in order to be able to find a causal relationship between a drug and an adverse reaction. The parameters used in identifying the signal pairs are really a vague, subjective measure rather than an objective measure. Up to our knowledge, no set of rules was clearly mentioned in the literature that took advantage of this vagueness. Furthermore, physicians often disagree one another owing to their knowledge and experience levels and usually there is no "ground truth" to indicate which physician is right or wrong. The developed fuzzy inference engine also uses the real patient databases. Such databases have not been used before in the literature for such purposes. Databases in current systems are mainly used to know the medical history of patients by only their physicians, improving the quality of provided services and reducing the costs of medical errors. These databases provide valuable information about patients, including age, sex, medication took by the patients, symptoms appears in patients, laboratory test results, and procedure followed by the physician at the visit time. The proposed ADR signal pairs detection methodology is based on five cues: temporal association, rechallenge, dechallenge, abnormality in laboratory tests and other explanation. The cues represent the higher-level information that is obtained from the patients' elementary data. The detection rules that use the above cues were acquired 
through the joint efforts of the engineering and medical team members.

Optimizing fuzzy rule weights and fuzzy sets parameters using genetic algorithm (GA) have been used in this paper to improve the initial performance of decision trees. The final decision tree performance has been enhanced with the achieved optimized fuzzy rule weights and fuzzy sets parameters. Using Waikato environment for knowledge analysis (WEKA) software [32], the collected and preprocessed training set are randomly chosen from real patients' dataset, and are used to build the decision tree model. After that, the developed model is tested on another portion of the data unseen in the training stage for model evaluation.

The rest of the paper is organized in the following manner: section 2 explains the framework of the proposed method. Results have been presented in section 3. Section 4 shows the result discussion and analysis. Finally the concluding remarks were given in section 5 .

\section{Materials and methods}

Figure 2 shows decision tree-based ADRs detection system. ADRs detection is characterized by a number of factors related to physician's experience.

In this paper ADRs detection is based on temporal association, dechallenge and rechallenge of a medication of interest, abnormality of a laboratory test, medication side effect risk, medications, and morbidities. To get some of those cues, we used the international classification of diseases, ninth edition, clinical modification (ICD-9) and physicians' current procedural terminology codes (CPT). These are two widely used coding standards in the USA. Using these codes, every clinical condition of the patients, symptoms that appear one patient and any treatment required or done by a health provider has a unique code. The ICD-9 code provides codes to classify diseases and a wide variety of signs, symptoms, abnormal findings, complaints, social circumstances, and external causes of injury or disease. Every health condition is assigned a unique category and given a unique code. For example, if a patient is diagnosed with Hepatitis C, he/she will be given the ICD-9 code "070.51". If the diagnosis is for something acute, something that goes away with treatment like a rash or the flu, then the ICD-9 code will be less important because the illness or condition will go away. However, if the patient is diagnosed with a chronic or lifelong problem, like heart disease or diabetes, the ICD-9 code will be more important and will affect his future medical care.

Since different ICD-9 codes may represent the same (or similar) diagnoses, we clustered the ICD-9 codes into a manageable number of categories. This was done based on the clinical classification system (CCS) for the ICD-9 fact sheet. CCS is developed by the Agency for Healthcare Research and Quality (AHRQ) [33]. The CCS can group over 13,600 ICD9 codes into 285 mutually exclusive and clinically meaningful categories. The clinical classification system makes it easier to quickly understand patterns of diagnoses and procedures. The physicians can easily analyze patient cases using CCS and assign them labels.

Temporal association describes the symptom duration which is the time between taking a drug and the appearance of symptoms. It should be noted that in the case of a potential ADR, exposure to a drug should always precede the effect (symptom). This distinction is important because the effect might result from entirely different causes (e.g., underlying diseases or reception of another medication). Dechallenge refers to the relationship between the time of discontinuity of a drug and abatement of a symptom which is disappearance duration. We cannot directly evaluate dechallenge of a pair since the drug stop date is usually unavailable in electronic health databases. However, we can indirectly assess the existence of dechallenge of a pair if a symptom occurs after the drug start date and another drug in the same class was prescribed after the appearance of the symptom. This is because the physicians often stop a drug and prescribe another drug in the same class to avoid the apparent adverse effect found in a patient. Rechallenge depicts the relationship between re-introduction of the drug discontinued before and recurrence of a symptom. Rechallenge is determined by the temporal associations of the two consecutive occurrences of the same pair one after taking the medication and the other one after the reintroduction of the medication. 


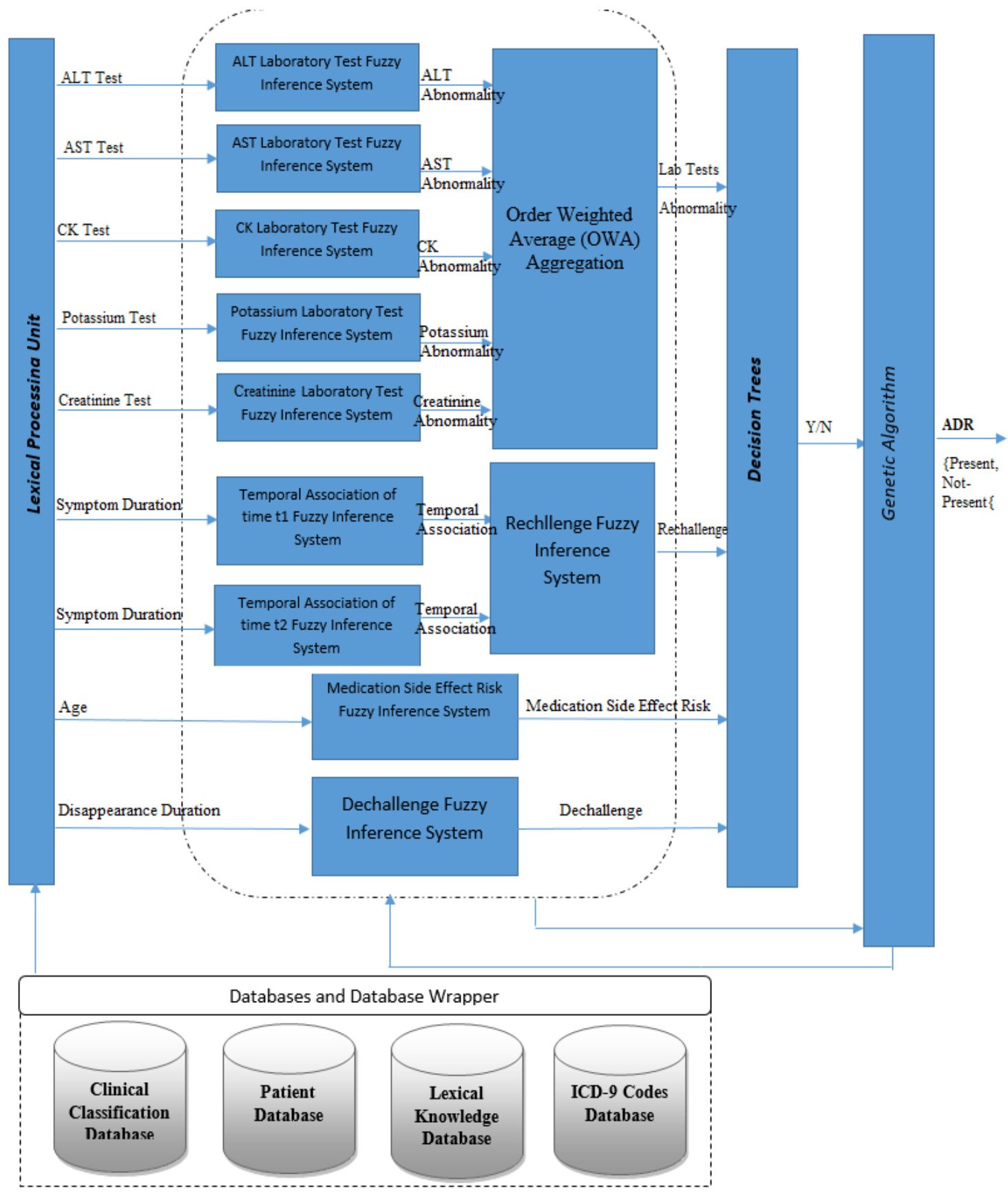

Figure 2 General architecture of the developed system

Functionally speaking, the proposed system architecture consists of four components, namely fuzzy inference system unit, decision tree unit, GA unit and database wrapper. The proposed system algorithm is shown in Figure 3.

Abnormality of a laboratory test shows the degree of elevation of a laboratory test result. The abnormality will be calculated for transaminases aspartate 114 aminotransferase (AST), alanine aminotransferase (ALT), creatine kinase (CK), potassium, and creatinine, which are common tests. Medication side effect risk gives the side effect risk degree that could be on a patient based on his/her age [34]. The side effect risk of a medication in a healthy adult is not that dangerous as in elderly patients. Medication factor shows the overall view of medications took by the patients, including medication name, class and quantity. 


\section{Training Phase:}

Retrieve training patients' demographic data (i.e. laboratory test results, age, medications, etc.)

Extract medical cues using fuzzy inference systems which represent the higher level information

(dechallenge, rechallenge, medication side effect risk, Laboratory test Abnormality)

Classify medical cues as present or not present ADR using decision tree

Optimize fuzzy rules weights and fuzzy sets parameters using genetic algorithm and update the

constructed decision tree

End by returning the optimized fuzzy rules weights and fuzzy sets parameters

\section{Testing Phase:}

Retrieve Testing patients' demographic data (i.e. laboratory test results, age, medications, etc.)

Extract medical cues using fuzzy inference systems which represent the higher level information

(dechallenge, rechallenge, medication side effect risk, Laboratory test abnormality)

Classify medical cues as present or not present ADR using decision tree

End by labelling the medical cases as present or not present ADR

Figure 3 Proposed system algorithm

The morbidity factor presents all the symptoms appear in a patient and have been verified by his physician. The symptoms are recorded at the time of physician visit as ICD-9 codes.

The medication plays a key role in identifying ADR since an adverse event can change by changing the medication. Sometimes the resulted adverse events are pleasant as in the case of some out of counter medications such as pain relievers and fever reducers. However, sometimes, the resulted adverse events are dangerous and can cause a great harm to the human body. In this paper a strategy of classifying drugs available in the market is used and has been embedded in our framework. The medications were catalogued according to the anatomical therapeutic chemical (ATC) classification [35]. This system is recommended by the WHO for drug utilization studies.

In the ATC classification system, the active substances are divided into different groups according to the organ or the system on which they act and their therapeutic, pharmacological and chemical properties. For example, in the ATC system medications captopril and enalapril, which are angiotensin converting enzyme (ACE) inhibitor medications are given the code C09AA.

\subsection{Fuzzy inference system unit}

Based on the above-mentioned factors, we defined input variables of Fuzzy Inference Engines, as shown in Table 1. Each variable is fuzzified by input fuzzy sets whose names, types, and parameters are specified in Table 1 . The mathematical type definitions are given in Table 2. Likewise, we define output variables and their fuzzy sets for the engine (Table 3). The linguistic concepts, most of which are inherently vague, are represented and manipulated by fuzzy sets using the theoretical tools provided by fuzzy set theory. They enable us to express and deal with various relations and functions that involve linguistic concepts. The construction of fuzzy sets involves a specific knowledge domain of interest, two experts in the adverse drug reaction domain and a knowledge engineer. The knowledge of, interest is elicited from the experts by the knowledge engineer. In the first stage the knowledge engineer attempts to elicit the knowledge in terms of propositions expressed in natural languages. In the second stage, he attempts to determine the meaning of each linguistic term employed in these propositions. During the second stage the functions representing fuzzy sets and operations are constructed. A direct method of construction has been used in this research. In direct methods, experts are expected to give answers to questions of various kinds that explicitly lead to construction of membership functions.

Fuzzy inference engine is equipped with the fuzzy rules that link the input variables to the output variables (Table 4). The initial rules and formulas are provided by the physicians on the team. There is a total of 40 rules developed by the physicians and is used in this paper. In this paper, we assign a confidence level, a value in $[0,1]$, to each rule based on the experience of the agent in that rule. The confidence levels affect the contribution of the rules which consequently affects the calculation of the output of the fuzzy inference engines. The inferred output of each rule is scaled by the confidence level value via algebraic product before aggregating the output of individual rules. A reference patient case is evaluated by the proposed system using the rules stored in the local patient database. 
Mansour

Table 1 Input variables and their fuzzy set names, types and parameters

\begin{tabular}{|c|c|c|c|c|c|}
\hline \multirow[t]{2}{*}{ Output variable } & \multirow[t]{2}{*}{ Fuzzy set name } & \multirow[t]{2}{*}{ Fuzzy set type } & \multicolumn{3}{|c|}{ Fuzzy set parameters } \\
\hline & & & $\mathbf{a}$ & $\mathbf{b}$ & c \\
\hline \multirow{3}{*}{ Temporal association } & Unlikely & \multirow{3}{*}{ Triangular } & - & 0 & 0.5 \\
\hline & Possible & & 0 & 0.5 & 1 \\
\hline & Likely & & 0.5 & 1 & - \\
\hline \multirow{3}{*}{ Dechallenge } & Unlikely & \multirow{3}{*}{ Triangular } & - & 0 & 0.5 \\
\hline & Possible & & 0 & 0.5 & 1 \\
\hline & Likely & & 0.5 & 1 & - \\
\hline \multirow{3}{*}{ Rechallenge } & Unlikely & \multirow{3}{*}{ Triangular } & - & 0 & 0.5 \\
\hline & Possible & & 0 & 0.5 & 1 \\
\hline & Likely & & 0.5 & 1 & - \\
\hline \multirow{5}{*}{ AST abnormality } & Very Low & \multirow{5}{*}{ Triangular } & - & 0 & 0.25 \\
\hline & Low & & 0 & 0.25 & 0.5 \\
\hline & Medium & & 0.25 & 0.50 & 0.75 \\
\hline & High & & 0.50 & 0.75 & 1 \\
\hline & Very High & & 0.75 & 1 & - \\
\hline \multirow{5}{*}{ ALT abnormality } & Very Low & \multirow{5}{*}{ Bell } & 0 & 0.13 & 3 \\
\hline & Low & & 0.25 & 0.13 & 3 \\
\hline & Medium & & 0.5 & 0.13 & 3 \\
\hline & High & & 0.75 & 0.13 & 3 \\
\hline & Very High & & 1 & 0.13 & 3 \\
\hline \multirow{4}{*}{ CK abnormality } & Very Low & \multirow{4}{*}{ Bell } & 0 & 0.17 & 3 \\
\hline & Low & & 0.33 & 0.17 & 3 \\
\hline & High & & 0.67 & 0.17 & 3 \\
\hline & Very High & & 1 & 0.17 & 3 \\
\hline \multirow{3}{*}{ Potassium abnormality } & Low & \multirow{3}{*}{ Gaussian } & 0 & 0.21 & - \\
\hline & Medium & & 0.5 & 0.21 & - \\
\hline & High & & 1 & 0.21 & - \\
\hline \multirow[t]{2}{*}{ Creatinine abnormality } & Low & \multirow[b]{2}{*}{ Bell } & 0 & 0.5 & 2.5 \\
\hline & High & & 1 & 0.5 & 2.5 \\
\hline \multirow[t]{4}{*}{ Medication side effect risk } & Very Low & \multirow{4}{*}{ Gaussian } & 0 & 0.14 & - \\
\hline & Low & & 0.33 & 0.14 & - \\
\hline & High & & 0.67 & 0.14 & - \\
\hline & Very High & & 1 & 0.14 & - \\
\hline
\end{tabular}

Table 2 Definitions of the fuzzy sets

Fuzzy set type

Triangular

Bell

Gaussian

\section{Fuzzy set definition}

$\mu_{T}(x)= \begin{cases}-\frac{1}{a-b}(a-x), & a \leq x \leq b \\ \frac{1}{c-b}(c-x), & b \leq x \leq c \\ 0, & \text { otherwise }\end{cases}$

$\mu_{B}(x)=\frac{1}{1+\left|\frac{a-x}{b}\right|^{2 c}}, \quad c>0$

$\mu_{G}(x)=\mathrm{e}^{-\frac{(a-x)^{2}}{2 b^{2}}}$

Table 3 Output variables and their fuzzy set names, types and parameters

\begin{tabular}{llllll}
\hline Input variable & Fuzzy set name & Fuzzy set type & \multicolumn{2}{c}{ Fuzzy set parameters } & C \\
\cline { 4 - 6 } & & & A & B & 15 \\
Symptom appearance & Short & \multirow{2}{*}{ Triangular } & 0 & 0 & 30 \\
& Medium & & 15 & 30 & -
\end{tabular}




\begin{tabular}{|c|c|c|c|c|c|}
\hline \multirow[t]{2}{*}{ Input variable } & \multirow[t]{2}{*}{ Fuzzy set name } & \multirow[t]{2}{*}{ Fuzzy set type } & \multicolumn{3}{|c|}{ Fuzzy set parameters } \\
\hline & & & $\mathbf{A}$ & B & $\mathbf{C}$ \\
\hline \multirow{3}{*}{ Disappearance duration } & Short & \multirow{3}{*}{ Triangular } & - & 0 & 15 \\
\hline & Medium & & 0 & 15 & 30 \\
\hline & High & & 15 & 30 & - \\
\hline \multirow{5}{*}{ AST test } & Very Low & \multirow{5}{*}{ Triangular } & - & 60 & 92.5 \\
\hline & Low & & 60 & 92.5 & 125 \\
\hline & Medium & & 92.5 & 125 & 157.5 \\
\hline & High & & 125 & 157.5 & 190 \\
\hline & Very High & & 157.5 & 190 & - \\
\hline \multirow{5}{*}{ ALT test } & Very Low & \multirow{5}{*}{ Bell } & 70 & 16 & 3 \\
\hline & Low & & 102.5 & 16 & 3 \\
\hline & Medium & & 135 & 16 & 3 \\
\hline & High & & 167.5 & 16 & 3 \\
\hline & Very High & & 200 & 16 & 3 \\
\hline \multirow{4}{*}{ CK test } & Very Low & \multirow{4}{*}{ Bell } & 150 & 75 & 3 \\
\hline & Low & & 300 & 75 & 3 \\
\hline & High & & 450 & 75 & 3 \\
\hline & Very High & & 600 & 75 & 3 \\
\hline \multirow{3}{*}{ Potassium test } & Low & \multirow{3}{*}{ Gaussian } & 5 & 0.42 & - \\
\hline & Medium & & 6 & 0.42 & - \\
\hline & High & & 7 & 0.42 & - \\
\hline \multirow[t]{2}{*}{ Creatinine test } & Low & \multirow[b]{2}{*}{ Bell } & 1.5 & 0.75 & 2.5 \\
\hline & High & & 3 & 0.75 & 2.5 \\
\hline \multirow{4}{*}{ Age } & Very Young & \multirow{4}{*}{ Gaussian } & 35 & 8 & - \\
\hline & Young & & 50 & 8 & - \\
\hline & Old & & 75 & 8 & - \\
\hline & Very Old & & 90 & 8 & - \\
\hline
\end{tabular}

Table 4 Fuzzy rules that link input and output variables

\begin{tabular}{|c|c|c|}
\hline Relationship between & Rules & $\begin{array}{l}\text { Confidence } \\
\text { Level }\end{array}$ \\
\hline $\begin{array}{l}\text { Symptom appearance and } \\
\text { temporal association }\end{array}$ & $\begin{array}{l}\text { - If Symptom Appearance is Short Then Temporal Association is Likely. } \\
\text { - If Symptom Appearance is Medium Then Temporal Association is Possible. } \\
\text { - If Symptom Appearance is Long Then Temporal Association is Unlikely. }\end{array}$ & $\begin{array}{l}\mathrm{CL}_{1} \\
\mathrm{CL}_{2} \\
\mathrm{CL}_{3}\end{array}$ \\
\hline $\begin{array}{l}\text { Disappearance duration } \\
\text { and dechallenge }\end{array}$ & $\begin{array}{l}\text { - If Disappearance Duration is Short Then Dechallenge is Likely. } \\
\text { - If Disappearance Duration is Medium Then Dechallenge is Possible. } \\
\text { - If Disappearance Duration is Long Then Dechallenge is Unlikely. } \\
\text { - If Symptom does disappear after withdrawal of the drug then Dechallenge is } \\
\text { Unlikely. }\end{array}$ & $\begin{array}{l}\mathrm{CL}_{4} \\
\mathrm{CL}_{5} \\
\mathrm{CL}_{6} \\
\mathrm{CL}_{7}\end{array}$ \\
\hline \multirow{5}{*}{$\begin{array}{l}\text { Temporal association and } \\
\text { rechallange }\end{array}$} & $\begin{array}{l}\text { - If Temporal Association of time } \mathrm{t}_{1} \text { is Likely and Temporal Association of } \\
\text { time } \mathrm{t}_{2} \text { is Likely Then Rechallenge is Likely. } \\
\text { - If Temporal Association of time } \mathrm{t}_{1} \text { is Likely and Temporal Association of } \\
\text { time } \mathrm{t}_{2} \text { is Possible Then Rechallenge is Likely. } \\
\text { - If Temporal Association of time } \mathrm{t}_{1} \text { is Likely and Temporal Association of } \\
\text { time } \mathrm{t}_{2} \text { is Unlikely Then Rechallenge is Possible. } \\
\text { - If Temporal Association of time } \mathrm{t}_{1} \text { is Possible and Temporal Association of }\end{array}$ & $\begin{array}{l}\mathrm{CL}_{8} \\
\mathrm{CL}_{9} \\
\mathrm{CL}_{10} \\
\mathrm{CL}_{11}\end{array}$ \\
\hline & $\begin{array}{l}\text { time } \mathrm{t}_{2} \text { is Likely Then Rechallenge is Likely. } \\
\text { - If Temporal Association of time } \mathrm{t}_{1} \text { is Possible and Temporal Association of } \\
\text { time } \mathrm{t}_{2} \text { is Possible Then Rechallenge is Possible. }\end{array}$ & $\mathrm{CL}_{12}$ \\
\hline & $\begin{array}{l}\text { - If Temporal Association of time } \mathrm{t}_{1} \text { is Possible and Temporal Association of } \\
\text { time } \mathrm{t}_{2} \text { is Unlikely Then Rechallenge is Possible. } \\
\text { - If Temporal Association of time } \mathrm{t}_{1} \text { is Unlikely and Temporal Association of }\end{array}$ & $\mathrm{CL}_{13}$ \\
\hline & $\begin{array}{l}\text { time } t_{2} \text { is Likely Then Rechallenge is possible. } \\
\text { - If Temporal Association of time } \mathrm{t}_{1} \text { is Unlikely and Temporal Association of }\end{array}$ & $\mathrm{CL}_{15}$ \\
\hline & $\begin{array}{l}\text { time } \mathrm{t}_{2} \text { is Possible Then Rechallenge is Possible. } \\
\text { - If Temporal Association of time } \mathrm{t}_{1} \text { is Unlikely and Temporal Association of } \\
\text { time } \mathrm{t}_{2} \text { is Unlikely Then Rechallenge is Unlikely. }\end{array}$ & $\mathrm{CL}_{16}$ \\
\hline
\end{tabular}




\begin{tabular}{|c|c|c|}
\hline Relationship between & Rules & $\begin{array}{l}\text { Confidence } \\
\text { Level }\end{array}$ \\
\hline $\begin{array}{l}\text { AST Test and AST } \\
\text { abnormality }\end{array}$ & $\begin{array}{l}\text { - If AST Test is Very Low Then AST Abnormality is Very Low. } \\
\text { - If AST Test is Low Then AST Abnormality is Low. } \\
\text { - If AST Test is Medium Then AST Abnormality is Medium. } \\
\text { - If AST Test is High Then AST Abnormality is High. } \\
\text { - If AST Test is Very High Then AST Abnormality is Very High. }\end{array}$ & $\begin{array}{l}\mathrm{CL}_{17} \\
\mathrm{CL}_{18} \\
\mathrm{CL}_{19} \\
\mathrm{CL}_{20} \\
\mathrm{CL}_{21}\end{array}$ \\
\hline $\begin{array}{l}\text { ALT Test and ALT } \\
\text { abnormality }\end{array}$ & $\begin{array}{l}\text { - If ALT Test is Very Low Then ALT Abnormality is Very Low. } \\
\text { - If ALT Test is Low Then ALT Abnormality is Low. } \\
\text { - If ALT Test is Medium Then ALT Abnormality is Medium. } \\
\text { - If ALT Test is High Then ALT Abnormality is High. } \\
\text { - If ALT Test is Very High Then ALT Abnormality is Very High. }\end{array}$ & $\begin{array}{l}\mathrm{CL}_{22} \\
\mathrm{CL}_{23} \\
\mathrm{CL}_{24} \\
\mathrm{CL}_{25} \\
\mathrm{CL}_{26}\end{array}$ \\
\hline $\begin{array}{l}\text { CK Test and CK } \\
\text { abnormality }\end{array}$ & $\begin{array}{l}\text { - If CK Test is Very Low Then CK Abnormality is Very Low. } \\
\text { - If CK Test is Low Then CK Abnormality is Low. } \\
\text { - If CK Test is High Then CK Abnormality in is High. } \\
\text { - If CK Test is Very High Then CK Abnormality is Very High. }\end{array}$ & $\begin{array}{l}\mathrm{CL}_{27} \\
\mathrm{CL}_{28} \\
\mathrm{CL}_{29} \\
\mathrm{CL}_{30}\end{array}$ \\
\hline $\begin{array}{l}\text { Potassium test and } \\
\text { potassium abnormality }\end{array}$ & $\begin{array}{l}\text { - If Potassium Test is Low Then Potassium Abnormality Low. } \\
\text { - If Potassium Test is Medium Then Potassium Abnormality is Medium. } \\
\text { - If Potassium Test is High Then Potassium Abnormality is High. }\end{array}$ & $\begin{array}{l}\mathrm{CL}_{31} \\
\mathrm{CL}_{32} \\
\mathrm{CL}_{33}\end{array}$ \\
\hline $\begin{array}{l}\text { Creatinine test and } \\
\text { creatinine abnormality }\end{array}$ & $\begin{array}{l}\text { - If Creatinine Test is Low Then Creatinine Abnormality is Low. } \\
\text { - If Creatinine Test is High Then Creatinine Abnormality is High. }\end{array}$ & $\begin{array}{l}\mathrm{CL}_{34} \\
\mathrm{CL}_{35}\end{array}$ \\
\hline $\begin{array}{l}\text { Age and medication side } \\
\text { effect risk }\end{array}$ & $\begin{array}{l}\text { - If Age is Very Young Then Medication Side Effect is Very Low. } \\
\text { - If Age is Young Then Medication Side Effect is Low } \\
\text { - If Age is Old Then Medication Side Effect is High. } \\
\text { - If Age is Very Old Then Medication Side Effect is Very High. }\end{array}$ & $\begin{array}{l}\mathrm{CL}_{36} \\
\mathrm{CL}_{37} \\
\mathrm{CL}_{38} \\
\mathrm{CL}_{39} \\
\mathrm{CL}_{40}\end{array}$ \\
\hline
\end{tabular}

Fuzzy inference engine evaluates the rules using the min-max fuzzy inference operations. The resulting aggregated fuzzy set is converted to numerical values for the output variables by Defuzzifier that uses the center of gravity scheme.

The ordered weighted averaging (OWA) operators provide a parameterized class of mean type aggregation operators such as the max, arithmetic average, median and mean, are members of this class. The OWA operator is a mean or averaging operator. This is a reflection of the fact that the operator is commutative, monotonic, bounded and idempotent. Different families of OWA operators can be used by choosing a different manifestation of the weighting factor.

The aggregated laboratory abnormality is calculated as a linear combination of the corresponding sub abnormalities. The aggregated laboratory abnormality is computed as follows:

Laboratory Abnormality = ALT Abnormality x w1 + AST Abnormality x w2 + CK Abnormality x w3 + Potassium Abnormality $\mathrm{x}$ w4 + Creatinine Abnormality x w5.

Where $w 1+w 2+w 3+w 4+w 5=1$
The weights control the importance of the sub abnormalities. In ADR problem some laboratory abnormalities are more important that the other based on the studied medication.

The agents are supported with ability of mapping free text terms of unique concepts. This was done by lexical processing unit. It uses the lexical variants generator (LVG) program provided by the National Library of Medicine [36]. LVG is the most powerful solution for lexical variations at the individual word level. This unit allows the agents to deal with inflectional variants, spelling variants, acronyms and abbreviations, expansions, derivational variants, synonyms as well as combinations of these. The Lexical Processing Unit uses Lexical Knowledge Base. This knowledge base typically contains linguistic knowledge, such as word meanings, the syntactic patterns in which they occur, and special usage and idiosyncratic information, organized around the words in the language.

\subsection{Decision tree unit}

ADR classification process as \{Present, Not Present involves four phases: data gathering phase, data preprocessing phase, the learning phase and the recognition phase. In data gathering, the training and test set will be obtained from medical databases. The 
second phase is to pre-process the experimental data, including data cleaning, sampling, creating new records (attributes) and record selection. The relatively less correlated and redundant records in the database will be removed in this phase. In the learning phase, the target is to build a model.

The last step is using the remainder of pre-processed data to test the model. A test set is used to determine the accuracy of the model. Usually, the given dataset is divided into training and test sets, with training set used to build the model and test set used to validate it (Figure 4).

A decision tree partition the input space of the dataset into mutually exclusive regions by giving each region a label $[27,37,38]$. The decision tree that consists of a root and internal nodes grows from a root node, by determining the best split that partition the region at internal nodes into disjoint smaller subsets and proceed down to the leaf node (terminal nodes) labelled as-present or not present. In order to do the split an error function that quantifies the performance of a node $t$ in separating data from different classes. The used error function is impurity function. The best known impurity function for splitting is entropy function and Gini index. By using the impurity function $\varphi$, the impurity measure of a given node $t$ as in (2).

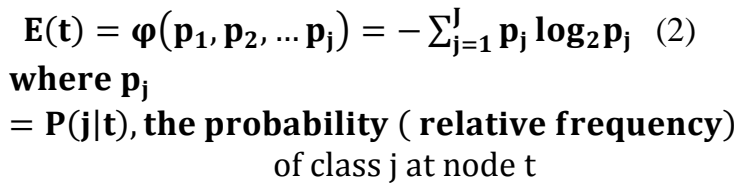$$
\mathbf{E}(\mathbf{t})=\varphi\left(\mathbf{p}_{1}, \mathbf{p}_{2}, \ldots \mathbf{p}_{\mathrm{j}}\right)=-\sum_{\mathrm{j}=1}^{\mathrm{J}} \mathbf{p}_{\mathrm{j}} \log _{2} \mathbf{p}_{\mathrm{j}}
$$

where $p_{j}$ $=\mathbf{P}(\mathbf{j} \mid \mathbf{t})$, the probability (relative frequency) of class $j$ at node $t$



Figure 4 The learning process: training and testing

The entropy function for $\mathrm{j}=3$ is shown in Figure 5 .

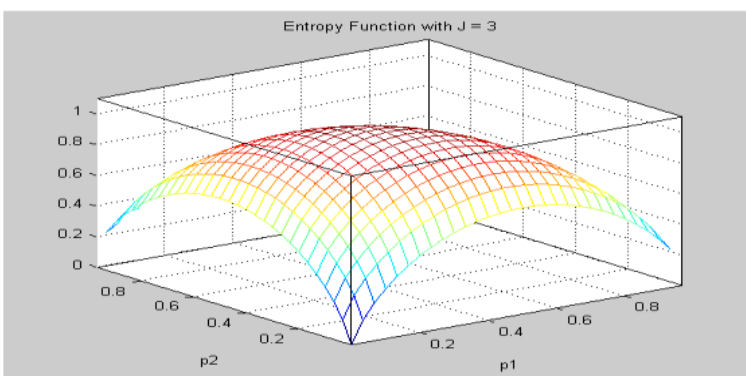

Figure 5 Entropy function with $\mathrm{J}=3$

The entropy measures homogeneity of a node. The maximum entropy value $\left(\log \mathrm{n}_{\mathrm{c}}\right.$ ) when records are evenly distributed among all classes, implying least information and minimum value of entropy (0.0) when all records belong to one class, implying most information.

Similarly, the impurity measure of a tree $\mathrm{T}$ can be expressed as

$E(T)=\sum_{t \in \Psi} \frac{n_{t}}{n} E(t)$

Where $\Psi$ is the set of terminal nodes in the tree $\mathrm{T}$, $\mathrm{n}_{\mathrm{t}}=$ number of records at child $\mathrm{t}$,

$\mathrm{n}=$ number of records at the terminal node.

The information gain is calculated as in (4)

$$
\begin{aligned}
& \text { GAIN }_{\text {Split }}=\mathrm{E}(\mathrm{P})-\mathrm{E}(\mathrm{T}) \\
& =\mathrm{E}(\mathrm{P})-\sum_{\mathrm{i}=1}^{\mathrm{k}} \frac{\mathrm{n}_{\mathrm{i}}}{\mathrm{n}} \mathrm{E}(\mathrm{i})
\end{aligned}
$$

Where Parent Node $\mathrm{P}$ (non-leaf node, node with partition) is split into k partitions (children), $\mathrm{n}_{\mathrm{i}}$ is the number of records in partition $\mathrm{i}, \mathrm{n}=$ number of records at the terminal node.

The information gain measures reduction in Entropy achieved because of the split. Choose the split that achieves most reductions (maximizes GAIN) due to the disadvantage that information gain tends to prefer splits that result in a large number of partitions, each being small but pure. GainRATIO is designed to overcome the disadvantage of Information Gain by adjusting information gain by the entropy of the partitioning.

$$
\text { GainRATIO }=\frac{\text { GAIN }_{\text {Split }}}{-\sum_{i=1}^{k} \frac{n_{i}}{n} \log _{2} \frac{n i}{n}}
$$

Where, $\mathrm{n}_{\mathrm{i}}$ is the number of records in partition $\mathrm{i}$

Four attributes with high information gain ranking are chosen to develop the decision tree model: challenge, rechallenge, a laboratory test abnormality, and medication risk factor. The algorithm for DT induction is shown in Figure 6. 


\section{Initialization}

Partition the training set space into \{Present ADR, Not-present ADR \}

Calculate the total Entropy of the system

Calculate the Gain ${ }_{\text {Split }}$ for each one of the five attributes \{Dechallenge, Rechallenge, Lab Test

Abnormality, Medication side effect risk\}

Choose the attribute with the highest information gain as the root node

\section{Expand the Tree starting from the previously selected node}

\section{Repeat until all records are classified}

Eliminate the previous selected node from the list of attributes

Partition attributes values of the remaining attributes in the space into \{Present, Not-present

Calculate the Entropy of the attributes

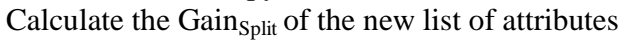

End by return Tree

Choose the attribute with the highest information gain as the next node

Figure 6 The decision tree induction algorithm

\subsection{Genetic algorithm unit}

$\mathrm{GA}$ is one of the most popular derivative free optimization technique which is based on the principles of evolution and natural genetics [38]. The GA starts by encoding each point in the parameter space into concatenated binary strings in which each concatenated value composed of set binary bits using binary coding techniques. Different binary encoding techniques are available such that Excess-3 code, binary coded decimal (BCD) and gray code. The resulted binary bits is called a chromosome. A set of chromosomes in the solution space is called a population. Chromosome consists of a set of genes that contains information about the key parameters in a candidate solution. Figure 7 shows a set of chromosomes of a population, where $\mathrm{n}$ weight being used as genes. Each gene is composed of m binary bits.

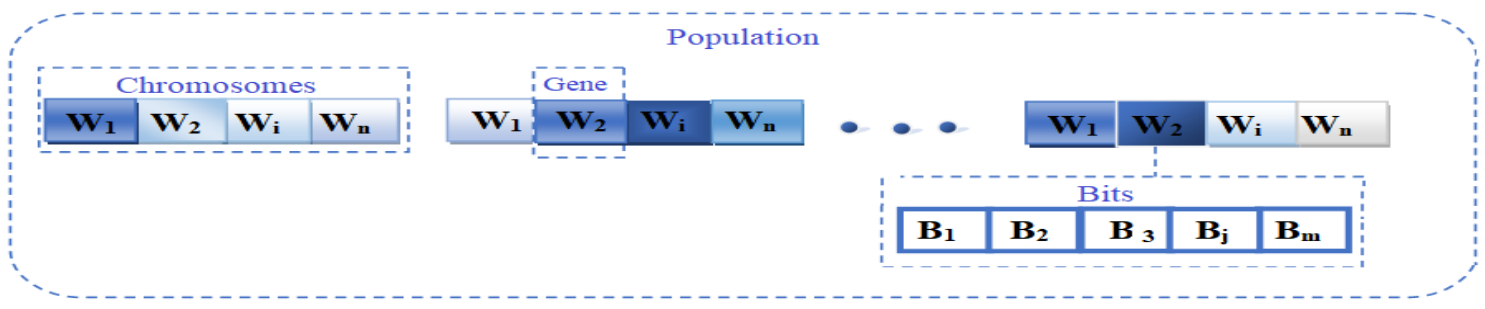

Figure 7 Block diagram of population structure

The second step after creating a population of chromosomes is to calculate the fitness value of each member of the population. The fitness value fi of the $\mathrm{i}^{\text {th }}$ weight parameter is the objective function evaluated at this weight set. The fitness function is chosen to be the root mean squared differences between the correct decision specified by physician $\mathrm{T}$ and the decision given by the decision tree $\widehat{T}$. The root mean squared error (RMSE) is given by (6). It is the standard deviation of the residuals (prediction errors). Residuals are a measure of how far from the actual class labeled by a physician,
Objective Function $=$ RMSE $=\sqrt{\frac{\sum_{\mathrm{i}=1}^{\mathrm{n}}(\mathrm{T}-\widehat{\mathrm{T}})^{2}}{\mathrm{n}}}(6)$

Where $\mathrm{n}$ is the number of records of training data

By this definition, the lower the fitness, the better the developed model and a fitness of zero means that the model achieve the desired behavior for all inputs. As long as the fitness measure ranks the individuals accurately based on their performance, the exact form of the fitness is irrelevant to the working of the algorithm. The proposed GA operates as illustrated in Figure 8. 


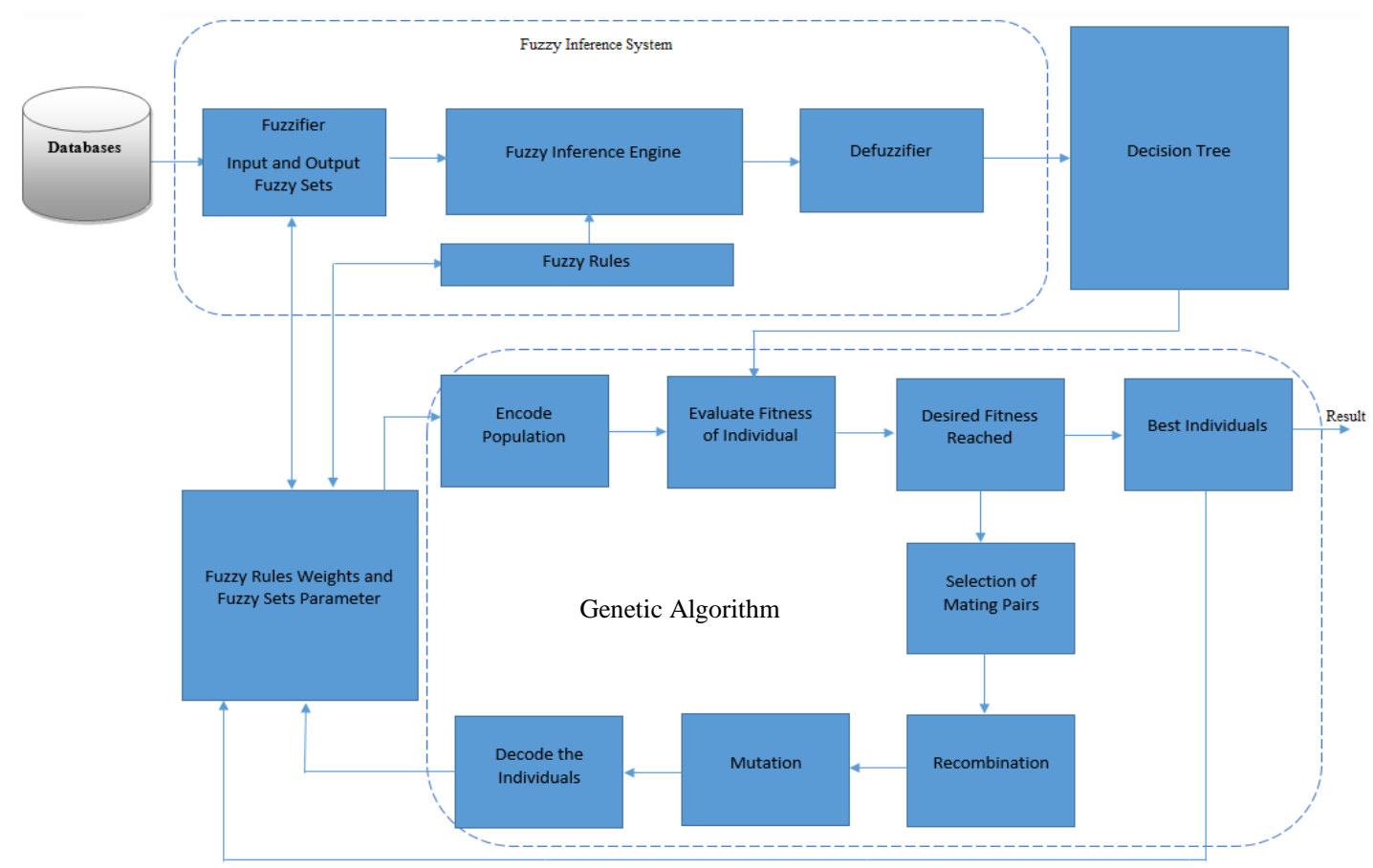

Figure 8 Block diagram of the developed GA methodology

GAs makes multiple way searches by creating a population of candidate solutions instead of just one single test solution. A starts by constructing a new population using genetic operation such as crossover and mutation through an iterative process until some convergence criteria are met.

The resulted new population will be decoded back to its original format. The process is:

1. Evaluation: Sort the population based on chromosomes scores (fitness).

2. Selection: Choose the best chromosomes to generate the next population (natural selection). $75 \%$ of the sorted population will be kept in the new population.

3. Crossover: Merge the chromosomes by mixing their genes. Repeat the crossover operation until the new population is fully generated.

4. Mutation: Change some chromosomes arbitrarily. Usually, around $1 \%$ of the crossover chromosomes will go through the mutation process. Mutation process prevents any single bit from converging to a value throughout the entire population.

A new generation is created by repeating the selection, recombination and mutation processes until all chromosomes in the new population replace the initial population. Figure 9 shows the algorithm used to determine the optimal solution.

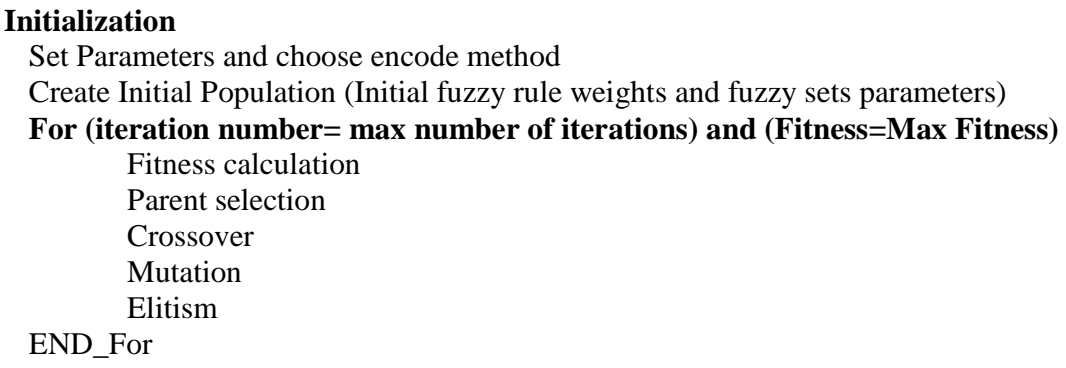

Decode individual with maximum fitness

End by return best solution: Output Fittest Fuzzy Rue Weights and Fuzzy Sets Parameters

Figure 9 GA-based decision tree optimization algorithm 
We retrieved the patients who received at least one of the 8 drugs of our interest in Veterans Affairs Medical Center in Detroit during the time period from January 1, 2005 to December 31, 2008. These 8 drugs represent the first targets in studying ADRs. These drugs are statin drugs and ACE drugs. A statin is a type of drug that helps patients lowers their cholesterol. An ACE is a type of drug that treats high blood pressure. The interested drugs include 6 statin drugs (i.e., rosuvastatin, atorvastatin, fluvastatin, lovastatin, pravastatin, and simvastatin) and $2 \mathrm{ACE}$ drugs (i.e., captopril, and enalapril).

The retrieved patient data includes dispensing of drug, office visits, symptoms experienced, and laboratory testing. For each event certain details were obtained. The total number of retrieved patients was 20,000 (19,102 males and 898 females). Their average age was 68.0. All the data stored in a Microsoft access database.

The retrieved patient data includes dispensing of drug, office visits, symptoms experienced, and laboratory testing. For each event certain details were obtained. The data for dispensing of drug includes the name of the drug, quantity of the drug dispensed, dose of the drug, drug start date, and the number of refills. The office visits data include treatment regimens, treatment start dates and stop dates. The symptoms experienced data includes the symptoms appearance date, the symptoms ICD-9 codes and the ICD-9 code description. The laboratory testing data includes the names of the laboratory tests, laboratory test dates, laboratory test normal ranges and laboratory test results.

The database had five tables, each of which contained one of the five types of information: (1) demographic data, (2) clinic visit data, (3) diagnostic data, (4) drug data, and (5) laboratory data.

The 20,000 patients clustered into three groups. The 1st group is for the patients who took only inhibitor drugs and they are 3,414 patients. The 2rd group is for the patients who took both drug classes, statin and inhibitor, and it contains 7,711 patients.
The 3rd group is for the patients who took only Statin drugs and it contains 8,875 patients. For the detection, evaluation experiments, we have selected randomly 200 patients out of the 20,000 patients ( $20 \%$ of the total population).

To improve the accuracy and decrease the training cost of the developed system, outlier and missing values in the data are removed. This decreases the population of 186 patients.

We have selected $1 \%$ patients from each group. From the 1st, 2nd, 3rd group we have selected 46, 60, and 80 patients respectively. The selection of $1 \%$ samples from each group was based on systematic sampling. The systematic sampling is a method of selecting samples from a larger population, according to a random starting point and a fixed, periodic interval. Typically, every "nth" member is selected from the total population to be included in the sampled population. The "nth" member is selected by dividing the total number of members in the general population by the desired number of members of the sampled population. For example, for selecting a random group of 88 patients from a population of 8,800 using systematic sampling, you would simply select every 100th person, since 8,800/88 $=100$. Systematic sampling can be considered random, as long as the periodic sampling interval is determined beforehand and the sampling starting point is random [39].

Two physicians were participated in this study. They were asked to independently review each of the 280 patient cases and checked whether the patient case suffer from ADR or not by assigning a \{present, not present\}. In this evaluation, patient cases were retrieved one by one from databases using a visual basic program done for that purpose (Figure 10).

If the best-fitting instances are selected with GA, this might lead to over-fitting. So, in order to make sure that there is no over fitting, 1/4 of the data was held ( 70 cases out of 280 cases) and will be used in testing the proposed algorithm. In all the experiments the GA operates with the configuration shown in Table 5. 


\begin{tabular}{|c|c|c|}
\hline Patient ID & AGE & \\
\hline \multicolumn{3}{|l|}{ PATIENT ICDg TABLE } \\
\hline ICD9 CODE & ICD9 DESCRIPTION & \\
\hline 305.90 & OTHER DRUG ABUSE-UNSPEC & C 1/11/2001 \\
\hline 303.93 & ALCOH DEP NEC/NOS-REMISS & S 1/1/8/2001 \\
\hline 311. & Depressive Disorder NOS (ICD-9-1 & \\
\hline 401.9 & HYPERTENSION NOS & 10/11/2004 \\
\hline 389.18 & SENSORINEURAL HEARING LO: & $0: 1 / 28 / 2005$ \\
\hline 389.8 & OTHER SPECIFIED FORMS OF I & $=11 / 28 / 2005$ \\
\hline 362.56 & EPIRETINAL MEMBRANE & $4 / 25 / 2005$ \\
\hline 362.11 & HYPERTENSIVE RETINOPATHY & Y 4/25/2005 \\
\hline 070.51 & Hepatitis C & $5 / 10 / 2005$ \\
\hline 304.20 & Cocaine Dependence, Nos & \\
\hline 304.00 & Opioid Dependence (ICD-9-CM 30 & 30 7/2/2007 \\
\hline \begin{tabular}{|l|l|l|}
$\operatorname{ann} n$ & Record: 1 of $12, M+$ |
\end{tabular} & 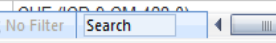 & \\
\hline \multicolumn{3}{|l|}{ PATIENT LAB TABLE } \\
\hline LAB DATE & TEST NMAE & \\
\hline APR 21,2005 02:22:21 & ALT & 64 \\
\hline APR 21,2005 02:22:21 & AST & 66 \\
\hline APR $21,200502: 22: 21$ & CKMB & 3.04 \\
\hline APR 21,2005 02:22:21 & CK & 337 \\
\hline APR 21,2005 02:22:21 & CREATININE & 1.2 \\
\hline APR 21,2005 02:22:21 & POTASSIUM & 4.1 \\
\hline APR $21,200506: 59: 25$ & CKMB & 1.79 \\
\hline APR 21,2005 06:59:25 & CK & 223 \\
\hline APR 28,2005 07:58:36 & ALT & 62 \\
\hline APR 28,2005 07:58:36 & AST & 62 \\
\hline APR 28,2005 07:58:36 & CREATININE & 1.1 \\
\hline APR 28,2005 07:58:36 & POTASSIUM & 3.4 \\
\hline APR 6,2005 14:27:56 & ALT & 40 \\
\hline APR 6,2005 14:27:56 & AST & 42 \\
\hline 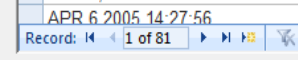 & $\begin{array}{l}\text { CRFATININF } \\
\text { No Filter Search }\end{array}$ & 0.9 \\
\hline \multicolumn{3}{|c|}{ Figure 10 User interface for showing patient dat } \\
\hline \multicolumn{3}{|l|}{ Table 5 GA setting } \\
\hline Parameter & Value & \\
\hline Population size & 20 & \\
\hline Variable range & $\begin{array}{l}{[1, \mathrm{~J}], \mathrm{J} \text { is a numl }} \\
\text { attributes }\end{array}$ & hber of \\
\hline Maximum generation & 200 & \\
\hline Crossover points & 2 points & \\
\hline Crossover probability & 0.75 & \\
\hline Mutation probability & 0.005 & \\
\hline Elitism & Yes & \\
\hline Selection method & Uniform selectio & \\
\hline
\end{tabular}

\section{Results}

Java was selected as the development language and J2SDK version 1.6.0_22 was used in the Java environment. Access database 2007 was adopted for the development of the database. Java database connectivity (JDBC), an application programming interface for the Java programming language, was used to access the database. JDBC could wrap a structured query language (SQL) statement, send it to the database, and retrieve the desired.

In this study, Weka is used [32] to construct decision trees, according to the training set with the standard algorithm J48 [37, 38]. Weka is a popular suite of

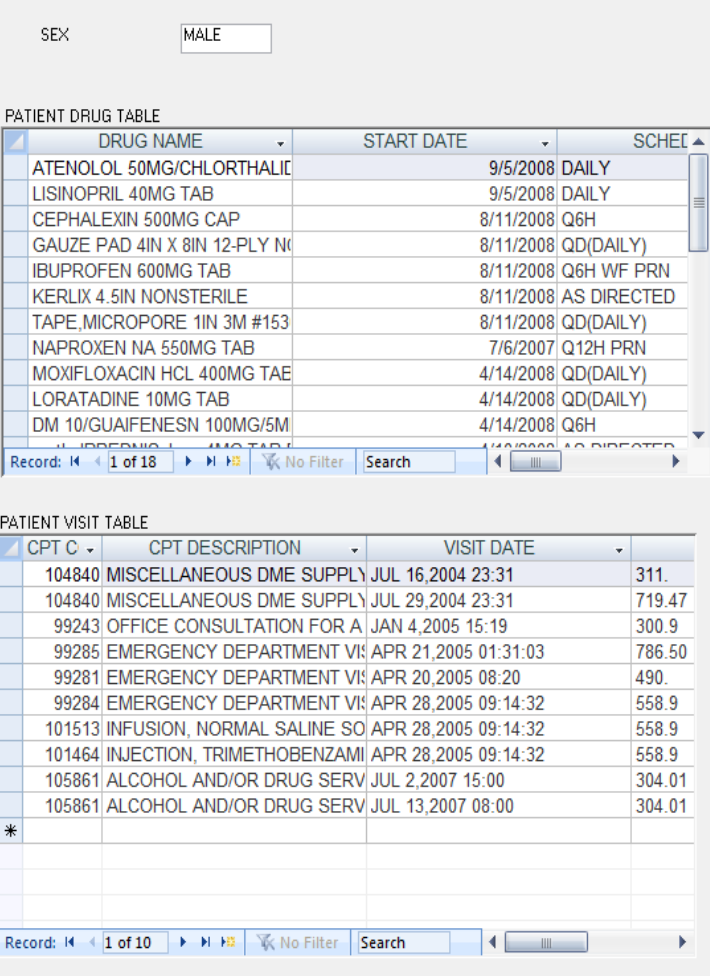

machine learning software written in Java, developed at the University of Waikato, New Zealand. Weka is a free software tool available under the GNU General Public License. It contains a collection of visualization tools and algorithms for data analysis and predictive modelling that support data preprocessing, clustering, classification, regression, visualization, and feature selection. Weka has a powerful graphical user interface that supports its functionality.

Once the features have been extracted and grouped into a feature vector, classification takes place, where each patient case is classified in one of the two classes: present or not present. J48 (C4.5) is an algorithm used to generate a decision tree. It has been developed by John Ross Quinlan. C4.5 is an extension of Quinlan's earlier ID3 algorithm [40]. The decision trees generated by $\mathrm{C} 4.5$ are normally used for classification.

It has been ranked first in the top 10 algorithms in data mining algorithm [41]. Figure 11 shows Weka J48 algorithm results GUI. Figure 12 is the Weka constructed decision tree from the Fuzzy Inference system optimized by GA. 
Mansour

Weka Explorer

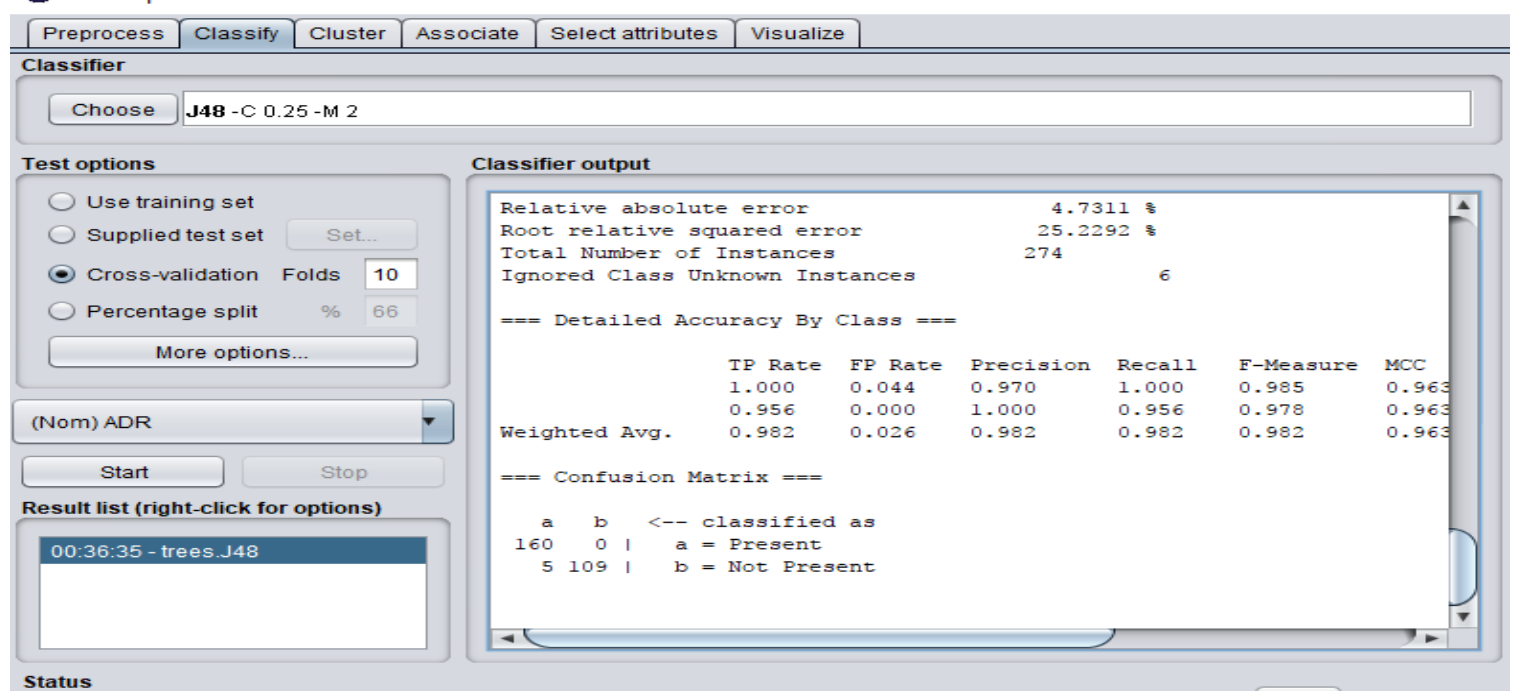

Figure 11 Weka J48 algorithm results

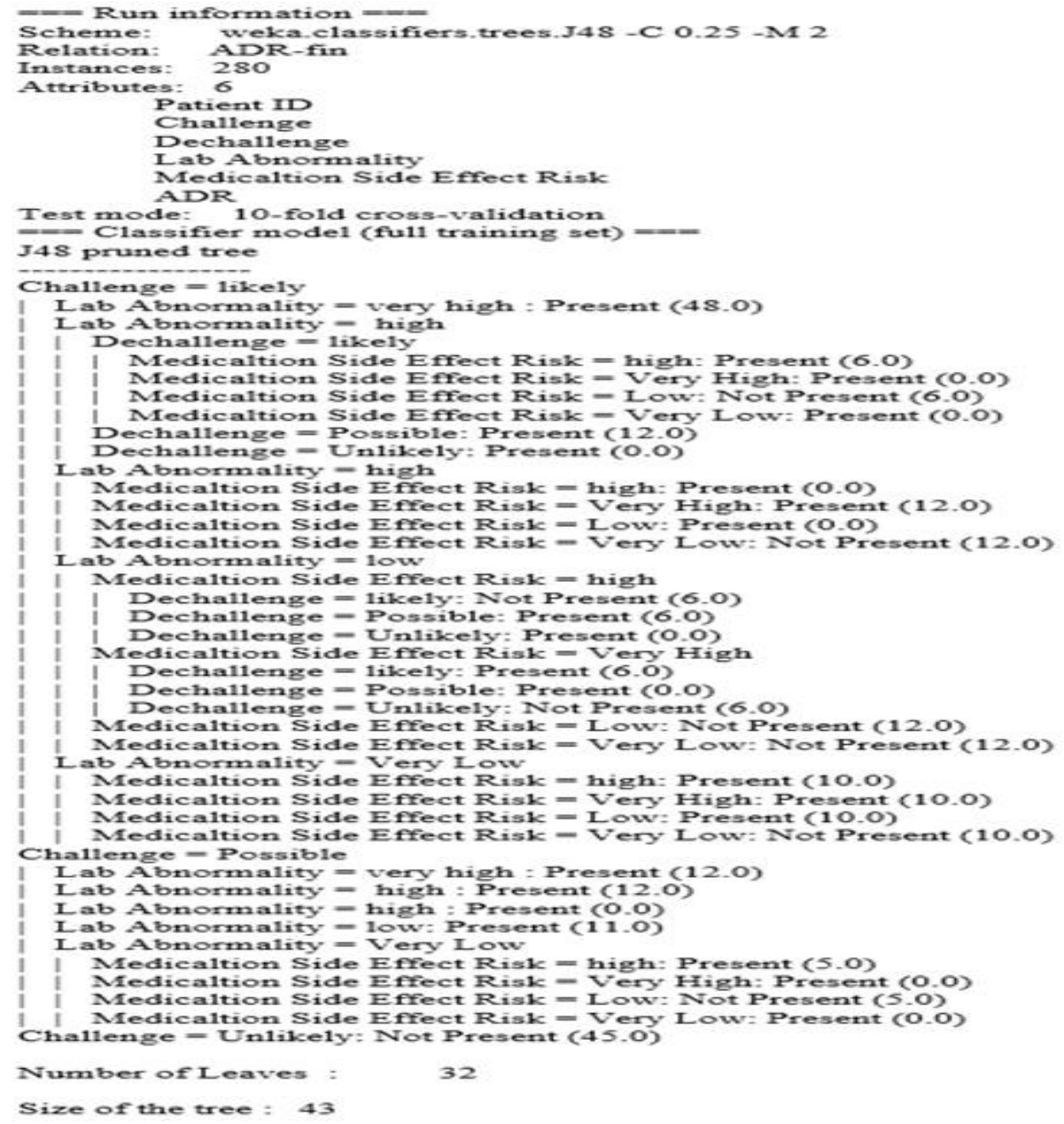

Figure 12 ADRs classification model 


\section{Discussion and analysis}

We examined the agreement between the results generated by the developed system and the one by the physicians.
We constructed the confusion matrix for each class (present or not -present). The confusion matrix has the form shown in Table 6.

Table 6 Confusion matrix

\begin{tabular}{llll}
\hline & Predicted class & & \\
\hline Actual & & Class=Present & Class=Not-Present \\
class & Class=Present & True Positive (TP) & False Negative (FN) \\
& Class=Not-Present & False Positive (FP) & True Negative(TN) \\
\hline
\end{tabular}

The performance measurements used in this paper were recall, precision, classifier $F_{1}$ rating, accuracy, pre-processing time, and running time. Pre-processing time includes dataset pre-processing time, and classifier training time. Running time indicates the classifier testing time.

They are defined as follows:

Recall (R) is the ratio of the relevant data among the retrieved. Precision $(\mathrm{P})$ is the ratio of the accurate data between the retrieved data. Their formulas are given as follows:

$\operatorname{Recall}(\mathbf{R})=\frac{\mathbf{T}_{\mathbf{P}}}{\mathbf{T}_{\mathbf{P}}+\mathbf{F}_{\mathbf{P}}}$ if $T P+F N>0$, otherwise undefined.

$\operatorname{Precision}(\mathbf{P})=\frac{\mathbf{T}_{\mathbf{P}}}{\mathbf{T}_{\mathbf{P}}+\mathbf{F}_{\mathbf{N}}} \quad$ if $T P+F N>0$, otherwise undefined.

The classifier $F_{1}$ rating is the harmonic mean of the classifier recall and the precision. It is given as

$\mathbf{F}_{\mathbf{1}}=\frac{2 * \mathbf{P} * \mathbf{R}}{\mathbf{P}+\mathbf{R}}$

Where $R$ represents the recall and $P$ represents the precision.

Accuracy, which indicates the fraction of correctly classified samples among all the samples, obtained by:

Accuracy $=\frac{T_{P}+T_{N}}{T_{P}+T_{N}+F_{P}+F_{N}}$

Dataset pre-processing time represents the time needed to read the dataset and convert it to an accepted format. Classifier training time represents the time needed by the classifier. Classifier testing time represents the time to classify the testing long string values.

Table 7 shows the resulted ADRs classification model evaluation results. The proposed DT model shows high accuracy on ADR classification (up to $98.17 \%$ ) on the test data. To further validate the results, K-fold cross validation was used. In K-fold the training set will be randomly split into $\mathrm{K}$ that has approximately the same size. Then the decision tree will be trained using (K-2) subsets. One of the two remaining subsets will be used for validation and the last for testing. This process will be repeated $K$ times, while a different subset is used for testing and validation.

Table 7 Overall performance results (training and validation set)

\begin{tabular}{ll}
\hline Total number of instances & 210 \\
\hline Correctly classified instances & 207 \\
& $98.5714 \%$ \\
\hline Incorrectly classified instances & 3 \\
& $1.4286 \%$ \\
\hline Kappa statistic & 0.9703 \\
\hline Mean absolute error & 0.0273 \\
\hline Root mean squared error & 0.1199 \\
\hline Relative absolute error & $5.6443 \%$ \\
\hline Root relative squared error & $24.3688 \%$ \\
\hline
\end{tabular}

Another performance indicated by confusion matrix is shown in Table 8. This confusion matrix was built based on data testing. We constructed the confusion matrix for each class (Present, not Present). The confusion matrix has the form shown in Table 8 .

Table 8 Confusion matrix (training and validation set)

\begin{tabular}{lll}
\hline & Present & Not present \\
\hline Present & 124 & 0 \\
Not Present & 3 & 83 \\
\hline
\end{tabular}

The performance measurement results are shown in Table 9.

Table 9 ADRs classification results (training and validation set)

\begin{tabular}{llllll}
\hline $\begin{array}{l}\text { TP } \\
\text { Rate }\end{array}$ & $\begin{array}{l}\text { FP } \\
\text { Rate }\end{array}$ & Precision & Recall & $\begin{array}{l}\text { F- } \\
\text { Measure }\end{array}$ & Class \\
\hline 1 & 0.035 & 0.976 & 1 & 0.988 & Present \\
0.965 & 0.000 & 1 & 0.965 & 0.982 & $\begin{array}{l}\text { Not } \\
\text { Present }\end{array}$ \\
\hline
\end{tabular}


Using the held 70 cases not previously used in the training or cross validation. The achieved results are shown in Table 10 and Table 11.

Table 10 Overall performance results (testing set)

\begin{tabular}{ll}
\hline Total number of instances & 70 \\
\hline Correctly classified instances & 68 \\
& $97.1429 \%$ \\
\hline Incorrectly classified instances & 2 \\
& $2.8571 \%$ \\
\hline Kappa statistic & 0.9405 \\
\hline Mean absolute error & 0.0359 \\
\hline
\end{tabular}

\begin{tabular}{ll}
\hline Root mean squared error & 0.1572 \\
\hline Relative absolute error & $7.4114 \%$ \\
\hline Root relative squared error & $31.9186 \%$ \\
\hline
\end{tabular}

Table 11 Confusion matrix (testing set)

\begin{tabular}{lll}
\hline & Present & Not present \\
\hline Present & 41 & 0 \\
Not Present & 2 & 27 \\
\hline
\end{tabular}

The performance measurement result is shown in Table 12.

Table 12 ADRs classification results (testing set)

\begin{tabular}{llllll}
\hline TP Rate & FP Rate & Precision & Recall & F-Measure & Class \\
\hline 1 & 0.069 & 0.953 & 1 & 0.976 & Present \\
0.931 & 0.000 & 1 & 0.931 & 0.964 & Not-Present \\
\hline
\end{tabular}

The final performance of decision tree has been improved compared with the initial performance of decision tree shown in Table 13.

Table 13 Initial decision tree performance results

\begin{tabular}{ll}
\hline Total number of instances & 210 \\
\hline Correctly classified instances & 124 \\
& $59.0476 \%$ \\
\hline Incorrectly classified instances & 86 \\
& $40.9523 \%$ \\
\hline Kappa statistic & 0 \\
\hline Mean absolute error & 0.4135 \\
\hline Root mean squared error & 0.643 \\
\hline Relative absolute error & $90.5263 \%$ \\
\hline Root relative squared error & $123.9239 \%$ \\
\hline
\end{tabular}

We have utilized Kappa statistic to estimate the levels of agreement. The Kappa coefficient is an estimate of the agreement between two raters. Kappa scores range between 1 (complete agreement) and 0 . If the two raters randomly assign their ratings, however, they would sometimes agree just by chance. Kappa gives us a numerical rating of the degree to which this occurs. The calculation is based on the difference between how much agreement is actually present ("observed" agreement) compared to how much agreement would be expected to be present by chance alone ("expected" agreement). Not everyone would agree on the interpretation of Kappa. However, a commonly cited scale [42] suggested that there is excellent agreement for values of Kappa greater than 0.75 , poor agreement for values less than 0.4 and fair to good agreement for values between 0.40 and 0.75 .

Table 10 summarizes the number of matches between the decision of the system and the two physicians.

The estimate of agreements is as follows: Kappa = 0.89 for physician 1 and the model; Kappa $=0.91$ for physician 2 and the model; Kappa $=0.88$ for physician 1 and physician 2. These coefficients suggest excellent agreement between the system and the physicians. The asymptotic standard error (ASE) is also computed, as well as $95 \%$ confidence bounds. The results of these two methods are shown in Table 14.

Table 14 Statistics for weighted Kappa, ASE and 95\% confidence limits

\begin{tabular}{llll}
\hline & Weighted Kappa coefficient & ASE & 95\% confidence limits \\
\hline Physician 1 vs. developed System & 0.89 & 0.044 & $(0.89,0.92)$ \\
Physician 2 vs. developed System & 0.91 & 0.034 & $(0.86,0.93)$ \\
Physician 1 vs. Physician 2 & 0.88 & 0.051 & $(0.85,0.89)$ \\
\hline
\end{tabular}

These coefficients suggest good to excellent agreement between the proposed model and the physicians. According to this experiment, the system showed a superior performance and it was able to solve the problem efficiently.

\section{Conclusion}

Several thousands of drugs are currently available on the market. A complete understanding of the safe use of drugs is not possible at the time when the drug is developed or marketed. Early detection of unknown 
adverse drug reactions could save lives and prevent unnecessary hospitalizations. The majority of current methods largely rely on spontaneous reports which suffer from serious underreporting, latency, and inconsistent reporting. Thus, they are not ideal for rapidly detect adverse drug reactions. In this paper, we have developed a system to detect adverse drug reaction using both fuzzy logic and decision tree approaches. Fuzzy logic is used to extract higher level information from patients' medical data. The extracted information is used by decision tree approach to construct a decision model.

Using real data of patients who were treated at the Veterans Affairs Medical Center in Detroit during the period between 2005 and 2008, the decision model has been constructed, tested and verified. The performance results show that the proposed system can detect adverse drug reaction with $97.14 \%$ accuracy. The experiment results were evaluated independently by two physicians on our team. Kappa statistics has been to evaluate the system results. The kappa coefficients show excellent agreement between the decision of physicians and the proposed model. In future work, other decision tree approaches such as random forests will be considered as they normally perform better than decision trees. Type 2 fuzzy sets or interval type 2 fuzzy can also be used instead of type 1 fuzzy sets in order to get a more precise decision and hence enhance the detection performance.

\section{Acknowledgment}

We would like to thank Ellison Floyd for retrieving the deidentified patient information from Detroit Veterans Affairs Medical Center's databases.

\section{Conflicts of interest}

The author has no conflicts of interest to declare.

\section{References}

[1] Edwards IR, Aronson JK. Adverse drug reactions: definitions, diagnosis, and management. The Lancet. 2000; 356(9237):1255-9.

[2] http://www.fda.gov/Drugs/GuidanceComplianceRegul atoryInformation/Surveillance/AdverseDrugEffects/uc m070461.htm. Accessed 26 January 2018.

[3] Stephens MD, Talbot J, Waller P. Stephens' detection of new adverse drug reactions. John Wiley \& Sons; 2004.

[4] Kaufman G. Adverse drug reactions: classification, susceptibility and reporting. Nursing Standard (2014+). 2016; 30(50):53-63.

[5] Svendsen K, Halvorsen KH, Vorren S, Samdal H, Garcia B. Adverse drug reaction reporting: how can drug consumption information add to analyses using spontaneous reports?. European Journal of Clinical Pharmacology. 2018; 74(4):497-504.

[6] Schmiedl S, Rottenkolber M, Szymanski J, Drewelow B, Siegmund W, Hippius M, et al. Preventable ADRs leading to hospitalization-results of a long-term prospective safety study with 6,427 ADR cases focusing on elderly patients. Expert Opinion on Drug Safety. 2018; 17(2):125-37.

[7] Dormann H, Criegee-Rieck M, Neubert A, Egger T, Geise A, Krebs S, et al. Lack of awareness of community-acquired adverse drug reactions upon hospital admission. Drug Safety. 2003; 26(5):353-62.

[8] Zheng Y, Lan C, Peng H, Li J. Using constrained information entropy to detect rare adverse drug reactions from medical forums. In annual international conference of the engineering in medicine and biology society 2016 (pp. 2460-3). IEEE.

[9] Cazacu I, Stroe R, Dondera R, Mogosan C, Haramburu F, Fourrier-Réglat A, et al. Adverse drug reactions of analgesic medicines: analysis of the Romanian pharmacovigilance database. Fundamental \& Clinical Pharmacology. 2018; 32(3):330-6.

[10] Stricker BH, Psaty BM. Detection, verification, and quantification of adverse drug reactions. BMJ. 2004; 329(7456):44-7.

[11] Klein E, Bourdette D. Postmarketing adverse drug reactions: a duty to report?. Neurology: Clinical Practice. 2013; 3(4):288-94.

[12] U.S. food and drug administration. https://open.fda.gov/data/faers/. Accessed 26 January 2018.

[13] U.K. Yellow Card ADR reporting system. http://yellowcard.mhra.gov.uk/. Accessed 26 January 2018.

[14] Canadian ADR reporting system. http://www.hcsc.gc.ca/dhp-mps/medeff/databasdon/index-eng.php. Accessed 26 January 2018.

[15] Central drugs standard control organization ADR reporting system of India. http://www.cdsco.nic.in/forms. Accessed 26 January 2018.

[16] Maggo SD, Chua EW, Chin P, Cree S, Pearson J, Doogue M, et al. A New Zealand platform to enable genetic investigation of adverse drug reactions. The New Zealand Medical Journal. 2017; 130(1466):62-9.

[17] Miller D. FDA's MedWatch program monitors drug safety, but it depends on nurses to report adverse events. ONS Connect. 2015; 30(3):49.

[18] Rehan HS, Chopra D, Holani SN, Mishra R. An observational study to compare the contents and quality of information furnished in CDSCO ADR reporting form, yellow card, medwatch and blue form by the healthcare professionals. International Journal of Risk \& Safety in Medicine. 2014; 26(1):1-8.

[19] Gupta A, Woosley R, Crk I, Sarnikar S. An information technology architecture for drug effectiveness reporting and post-marketing surveillance. International Journal of Healthcare Information Systems and Informatics (IJHISI). 2007; 2(3):65-80. 
[20] McGee S. Evidence-based physical diagnosis E-Book. Elsevier Health Sciences; 2016.

[21] Kelly M, Kaye KI, Davis SR, Shenfield GM. Factors influencing adverse drug reaction reporting in New South Wales teaching hospitals. Journal of Pharmacy Practice and Research. 2004; 34(1):32-5.

[22] Evans SJ, Waller PC, Davis S. Use of proportional reporting ratios (PRRs) for signal generation from spontaneous adverse drug reaction reports. Pharmacoepidemiology and Drug Safety. 2001; 10(6):483-6.

[23] Abin D, Mahajan TC, Bhoj MS, Bagde S, Rajeswari $\mathrm{K}$. Causal association mining for detection of adverse drug reactions. In international conference on computing communication control and automation 2015 (pp. 382-5). IEEE.

[24] Harpaz R, Chase HS, Friedman C. Mining multi-item drug adverse effect associations in spontaneous reporting systems. In BMC bioinformatics 2010 (p. S7). BioMed Central.

[25] Kubota K, Koide D, Hirai T. Comparison of data mining methodologies using Japanese spontaneous reports. Pharmacoepidemiology and Drug Safety. 2004; 13(6):387-94.

[26] Lilienfeld DE. A challenge to the data miners. Pharmacoepidemiology and Drug Safety. 2004; 13(12):881-4.

[27] Giannakopoulos I, Tsoumakos D, Koziris N. A decision tree based approach towards adaptive modeling of big data applications. In international conference on Big Data. 2017 (pp. 163-72). IEEE.

[28] Belohlavek R, Klir GJ. Concepts and fuzzy logic. MIT Press; 2011.

[29] Singhai R. Introduction to fuzzy logic paperback. PHI Learning; 2013.

[30] Zadeh LA. Fuzzy sets. Information and control. 1965; 8(3): 338-53.

[31] Orchard R. Fuzzy reasoning in JESS: the fuzzyj toolkit and fuzzyjess. International conference on enterprise information systems 2001 (pp. 533-42). National Research Council, Canada.

[32] University of Waikato. Weka Software. https://www.cs.waikato.ac.nz/ml/weka/. Accessed 26 January 2018.

[33] Doc N. Department of Health \& Human Services. 2004.

[34] Mansour AM. A multi-agent intelligent system for monitoring health conditions of elderly people. International Journal of Electrical, Robotics, Electronics and Communications Engineering. 2014; 8(6):993-8.
[35] U.S. food and drug administration, National Drug Code Directory, USA. http://www.fda.gov/Drugs/InformationOnDrugs/ucm1 42438.htm. Accessed 26 January 2018.

[36] U.S. National Library of Medicine. http://www.nlm.nih.gov. Accessed 26 January 2018.

[37] Olson D. Data mining models. Business Expert Press; 2016.

[38] Witten IH, Frank E, Hall MA, Pal CJ. Data mining: practical machine learning tools and techniques. Morgan Kaufmann; 2016.

[39] Granger CW, Siklos PL. Systematic sampling, temporal aggregation, seasonal adjustment, and cointegration theory and evidence. Journal of Econometrics. 1995; 66(1-2):357-69.

[40] Quinlan JR. C4. 5: programs for machine learning. Elsevier; 2014.

[41] Wu X, Kumar V, Quinlan JR, Ghosh J, Yang Q, Motoda $\mathrm{H}$, et al. Top 10 algorithms in data mining. Knowledge and Information Systems. 2008; 14(1):137.

[42] Landis JR, Koch GG. The measurement of observer agreement for categorical data. Biometrics. 1977; 33(1):159-74.

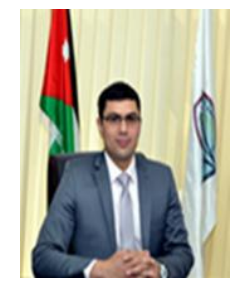

Dr. Ayman M Mansour received his $\mathrm{Ph} . \mathrm{D}$. degree in Electrical Engineering from Wayne State University in 2012. Dr. Mansour received his M.Sc degree in Electrical Engineering from University of Jordan, Jordan, in 2006 and his B.Sc degree in Electrical and Electronics Engineering from University of Sharjah, UAE, in 2004. He graduated top of his class in both Bachelor and Master. Currently, Dr. Mansour is an Assistant Professor in the Department of Communication and Computer Engineering, Tafila Technical University, Jordan. He is also the director of the Energy Research Center at Tafila Technical University. His areas of research include Communication Systems, Multiagent Systems, Fuzzy Systems, Data Mining and Intelligent Systems. He conducted several researches in his area of interest. Dr. Mansour is a member of IEEE, Michigan Society of Professional Engineers, IEEE Honor Society (HKN), Society of Automotive Engineers (SAE), Tau Beta $\mathrm{Pi}$ Honor Society, Sigma Xi and Golden Key Honor Society

Email: mansour@ttu.edu.jo 